\title{
Enhanced Signal Micro-Raman Study of SiGe Nanowires and SiGe/Si Nanowire Axial Heterojuntions Grown Using Au and Ga-Au Catalysts
}

\author{
J.Anaya , A. Torres, J.Jiménez, C. Prieto, A.Rodríguez, T.Rodríguez , C. \\ Ballesteros
}

\begin{abstract}
MicroRaman spectroscopy was used for the characterization of heterostructured $\mathrm{SiGe} / \mathrm{Si}$ nanowires. The NWs were grown with alloyed AuGa catalysts droplets with different Ga compositions aiming to make more abrupt heterojunctions. The heterojunctions were first characterized by TEM; then the NWs were scanned by the laser beam in order to probe the heterojunction. The capability of the MicroRaman spectroscopy for studying the heterojunction is discussed. The results show that the use of catalysts with lower Ge and Si solubility (AuGa alloys) permits to achieve more abrupt junctions.
\end{abstract}

\section{INTRODUCTION}

Group IV semiconductor nanowires (NWs) attract a great deal of attention because of its full compatibility with complementary metal oxide semiconductor (CMOS) technology. In particular, alloyed $\mathrm{SiGe}$ NWs present a great interest since they permit the continuous tunability of the physical properties by the intrinsic effect of the alloy composition, and are necessary for fabricating heterostructured group IV NWs essential for the electrically driven nanodevices [1]. The SiGe alloys have excellent properties for high frequency devices; moreover, SiGe NWs present excellent properties for thermoelectric conversion and light collection for energy applications. The control of the NW composition is a step forward to the fabrication of high quality NW heterostructures (HEs). In this way, one needs to select the optimal temperature and pressure conditions for the decomposition of the $\mathrm{Si}$ and Ge precursors, as well as the solubility of Si and Ge atoms in the catalysts droplets. The vapour liquid solid (VLS) method presents inherent difficulties for achieving abrupt heterointerfaces, due to the reservoir effect associated with the remaining $\mathrm{Si}$ or $\mathrm{Ge}$ solved in the catalysts droplet once the corresponding precursor gas is switched off [1-3]. In this way, the use of droplets in which the solubility of the atoms, either $\mathrm{Si}$ or $\mathrm{Ge}$, is lower shall benefit the $\mathrm{HE}$ abruptness. In this context, the use of alloyed AuGa catalysts droplets is expected to substantially narrow the trailing interface in $\mathrm{SiGe} / \mathrm{Si}$ NW HEs, because of the reduced solubility of $\mathrm{Ge}$ in $\mathrm{Ga}[2,3]$. The characterization of the NWs composition, and more specifically of the HEs, is a crucial issue for the optimization of the growth processes of axial HEs. MicroRaman spectroscopy is a powerful tool for studying group IV NWs. We present herein a microRaman study of $\mathrm{SiGe} / \mathrm{Si} \mathrm{NW}$ axial HEs grown by VLS with different $\mathrm{AuGa}$ droplet catalysts compositions, using a low pressure chemical vapour deposition (LPCVD); we discuss about the main issues concerning the characterization of the HEs by means of microRaman analysis. 


\section{EXPERIMENTAL}

$\mathrm{SiGe} / \mathrm{Si}$ axial NW HEs were grown by the vapour- liquid- solid (VLS) method using a commercial LPCVD. $\mathrm{Si}_{2} \mathrm{H}_{6}$ and $\mathrm{GeH}_{4}$ were used as precursor gases and alloyed $\mathrm{Ga}-\mathrm{Au}$ metal droplets of different compositions were used as catalysts. The nominal $\mathrm{Ga}$ content of the droplets was $0 \%$ (pure $\mathrm{Au}$ ), $50 \%$, and $90 \%$, for the details see [4]

Single Si NWs were grown at $470{ }^{\circ} \mathrm{C}$ using $\mathrm{Si}_{2} \mathrm{H}_{6}$ as a precursor; while the $\mathrm{SiGe}$ NWs (with a Ge atomic fraction close to 0.1 ) were grown at $430{ }^{\circ} \mathrm{C}$ using a $\mathrm{GeH}_{4}: \mathrm{Si}_{2} \mathrm{H}_{6}$ gas flow ratio, the carrier gas was $\mathrm{H}_{2}$ keeping a total pressure of $400 \mathrm{mTorr}$ all over the growth run [7]. Under these growth conditions NWs with an average diameter between 30 and $50 \mathrm{~nm}$, exhibiting good crystalline quality, were obtained. In a previous work, the different growth approaches followed to achieve a good matching between the SiGe and $\mathrm{Si}$ segments of the NW HEs were discussed. Switching off the GeH4 source, keeping the continuous flow of $\mathrm{Si} 2 \mathrm{H} 6, \mathrm{SiGe} / \mathrm{Si} \mathrm{NW} \mathrm{HE}$ were fabricated.

The NW HEs were separated from the substrate in an ultrasonic bath, and suspended in methanol. Transmission Electron Microscopy (TEM) was used for the characterization of the samples. Compositional studies were carried out in Z-contrast Scanning TEM (STEM) mode using an Energy Dispersive X-ray Emission (EDX) system attached to the microscope. Si and Ge EDX compositional maps were recorded to study the NW composition across the HE interface. High resolution TEM images were acquired for studying the crystalline quality of the HE.

MicroRaman spectroscopy was carried out on individual NWs deposited on a metallic substrate, either Al or Au evaporated on Si substrates. The Raman spectra were acquired along the NW, at low laser power excitation in order to avoid NW heating. The scanning line along the NW was acquired in steps of $\approx 10 \mathrm{~nm}$. The measurements were carried out with a Jobin Yvon UV-HR Labram Raman spectrometer, using a high magnification x100 objective with numerical aperture (NA) of 0.95 , and $532 \mathrm{~nm}$ laser excitation.

\section{TRANSMISSION ELECTRON MICROSCOPY (TEM)}

The Z-contrast STEM images did not reveal the presence of the junction in the NWs, because of the low Ge atomic fraction $(\approx 0.1)$. The junction was observed in the EDX maps. While the Si $\mathrm{K}_{\alpha}$ signal was almost homogeneous across the junction, the Ge $\mathrm{K}_{\alpha} \mathrm{X}$-ray signal revealed the HE. The characterization of the junction is difficult to achieve using these composition maps, since it does not provide absolute concentration values, and the composition profiles across the junction are fuzzy. However, it permits to see a more abrupt shift across the interface for the NWs grown from alloyed $\mathrm{Ga}-\mathrm{Au}$ catalyst droplets. Some of the NWs present a discontinuous Ge rich outer shell, which noises the composition images.

High resolution TEM images of the junction region of SiGe/Si HEs did not show distortions at the junction, stacking faults and other kind of defects are not present. A thin native oxide shell about 2-3nm thick was observed on the surface of the NWs. 


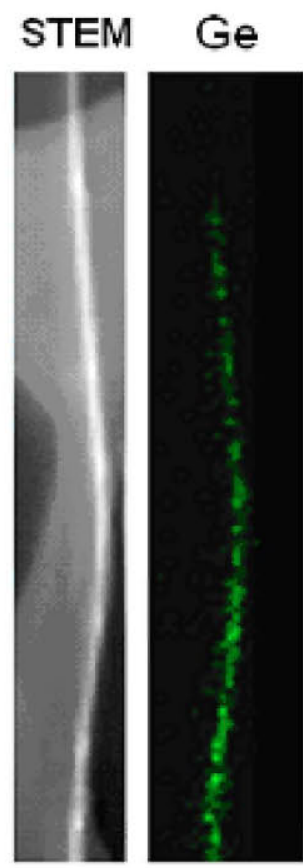

(a)

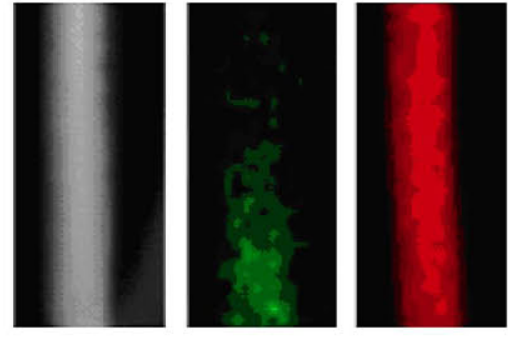

(b)
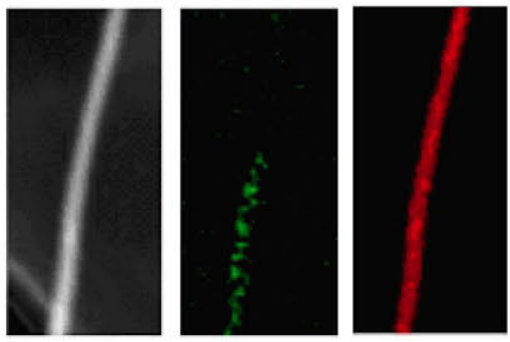

(c)

Figure 1. Z-contrast STEM images and EDX compositional maps obtained using the $\mathrm{Si} \mathrm{K}_{\alpha}$ and $\mathrm{Ge} \mathrm{K}_{\alpha} \mathrm{X}$-ray lines of $\mathrm{SiGe} / \mathrm{Si}$ trailing HEs grown with metal droplets of pure $\mathrm{Au}(\mathrm{a}), 50 \% \mathrm{Ga}(\mathrm{b})$ and $90 \% \mathrm{Ga}(\mathrm{c})$.
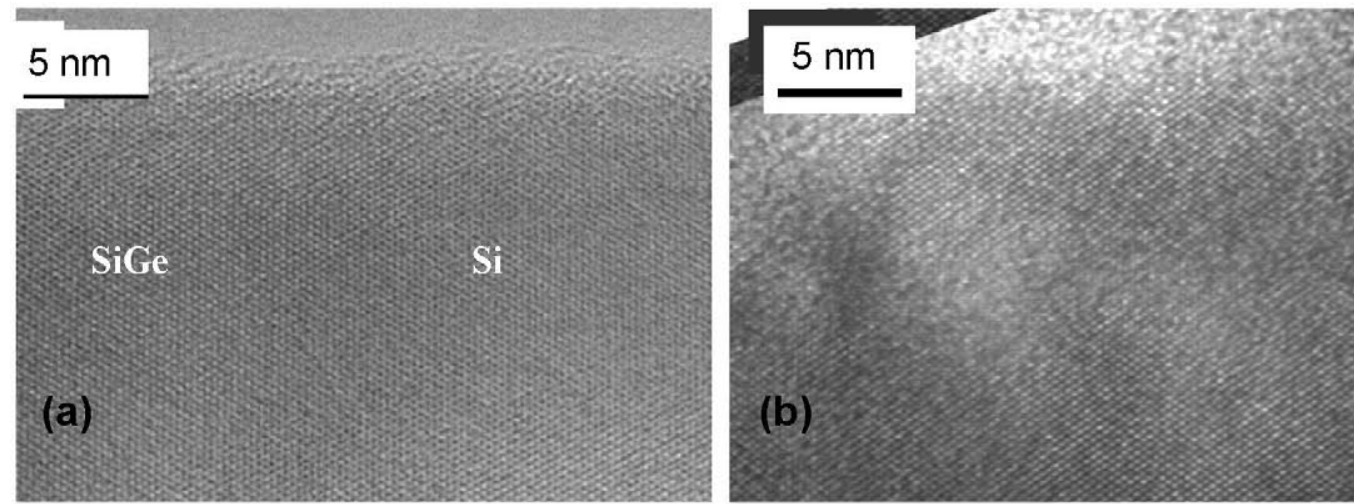

Figure 2. High resolution TEM micrographs of SiGe/Si NW HEs localized by EDX. Ga-Au with $50 \% \mathrm{Ga}$ (a) and $75 \% \mathrm{Ga}$ (b). The SiGe segment is on the left side of the images.

\section{RAMAN SPECTRUM OF NWS}

We afforded the capabilities of microRaman spectroscopy as a method to study the HE region of NWs. First, one should mention that the measurement of the Raman spectrum of individual NWs requires of experimental conditions that permit to neglect the temperature increase induced by the laser beam [5]. This can be achieved in conditions of electromagnetic amplification, under which the Raman signal is sharply enhanced [6], allowing to work under very low laser power excitation and negligible induced heating. For this purpose, the NWs were deposited on an $\mathrm{Au}$ or $\mathrm{Al}$ evaporated $\mathrm{Si}$ 
substrate [7]. The NWs deposited on these substrates were found to give resonance under excitation with a $532 \mathrm{~nm}$ laser beam polarized parallel to the NW axis. The Raman signal enhancement is diameter dependent. A series of resonances appear for specific NWs diameters; in particular a resonance was estimated for the diameter range of our NWs $(\approx 40 \mathrm{~nm})$. It should be noted that the resonance conditions should depend on the NW composition; therefore, Raman intensity differences between the two NW segments shall be observed depending on the NW diameter.
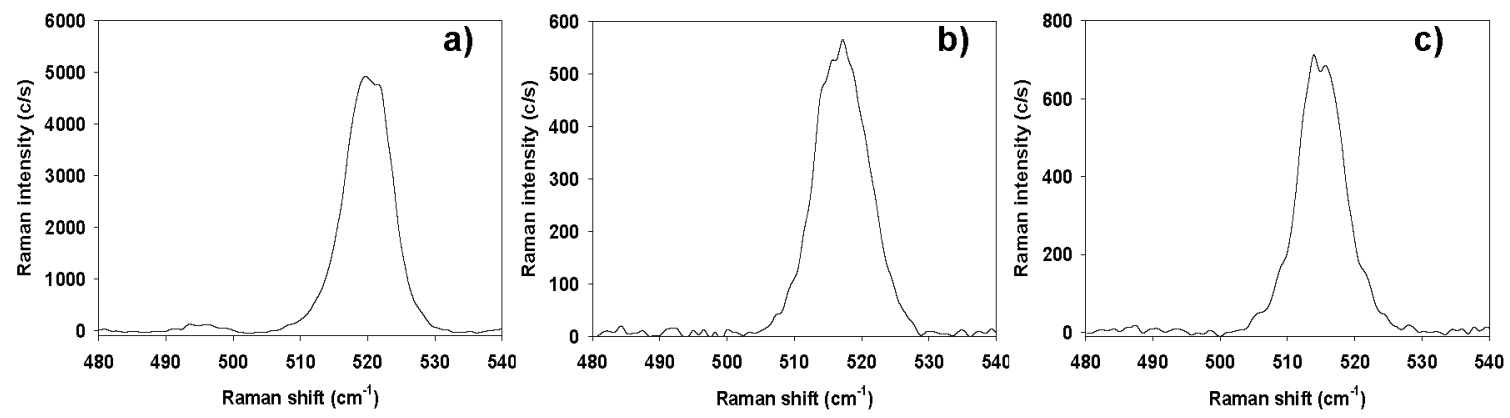

Fig.3. Raman spectra recorded in the Si segment (a), the HE region (b) and the SiGe segment (c).

Under resonance conditions, the Raman intensity per unit volume can be enhanced more than two orders of magnitude with respect to the Raman intensity per unit volume of the bulk material. This amplification will be crucial for the sensitivity of the Raman spectrum to the $\mathrm{HE}$, taking account of the very small scattering volume associated with HE.

The laser beam was scanned along the NW in steps of $10 \mathrm{~nm}$, so that the Raman spectrum is recorded along the full length of the NWs. Typical Raman spectra recorded along the NW grown with pure Au catalysts droplets are shown in Fig.3. The Ge fraction estimated from the Si-Si Raman band of the SiGe NW segment is $\approx 0.09$. One observes a significant signal intensity difference between the two NW segments, in this $\mathrm{NW}$, circa a factor 6 more intense for the $\mathrm{Si}$ segment, which can be explained by different resonance conditions on both NW segments. One also observes a change in the spectrum shape when crossing the HE. Here, we have to argue about whether the spectrum including the $\mathrm{HE}$ is the alone consequence of the convolution of the spectra of the two NW segments when the laser beam is sharing both, or there is a non negligible contribution from the NW HE. It should be noted that the laser beam diameter is $\approx 1 \mu \mathrm{m}$, which means that the contribution to the Raman scattering volume is much higher for the two NW segments than for the HE region, which is only tens of nms wide.

In order to answer this question one has to consider the Gaussian profile of the laser beam, and how it is shared by the two segments at a specified position of the laser beam with respect to the HE. The laser intensity Gaussian profile is represented in Fig.4; the vertical bar represents the position of the $\mathrm{HE}$, and the width of the bar corresponds to the width of the HE. The two shaded regions at both sides of the HE bar give the total laser intensity exciting each one of the two NW segments, either Si or SiGe. 


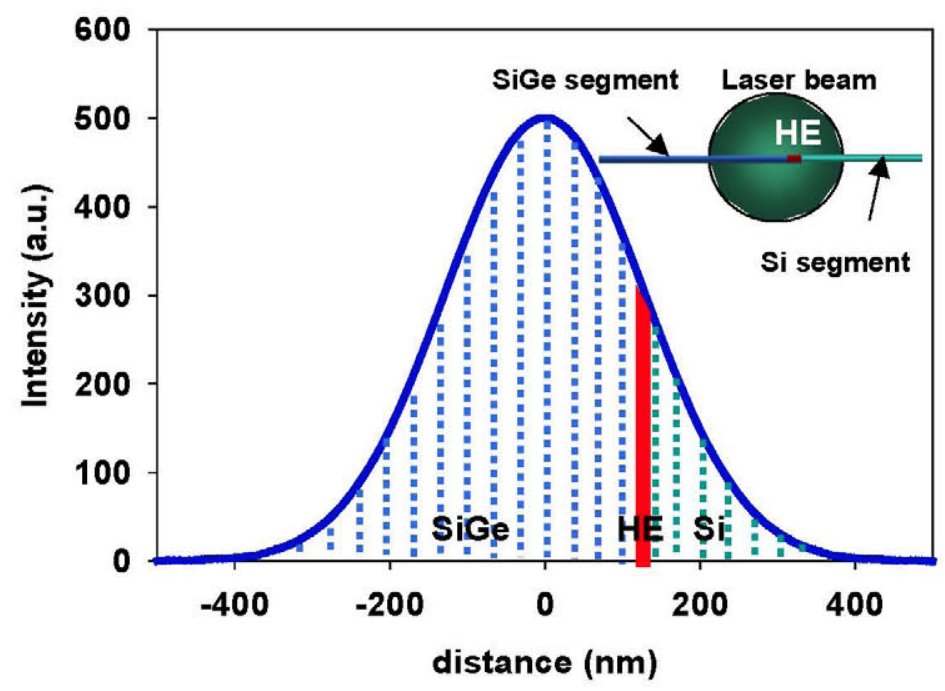

Fig.4. Gaussian laser intensity profile (blue line); the HE is represented by the red bar, the laser intensity exciting the HE is the area of the red bar. The shaded areas at both sides of the HE bar represent the laser fractions of the laser beam exciting the $\mathrm{SiGe}$ and Si NW segments respectively. The inset is a scheme of the NW inside the laser beam during the Raman measurement. The

In order to elucidate the role of the HE on the Raman spectrum we calculated the spectra for different positions of the laser beam with respect to the HE, taking different $\mathrm{HE}$ widths. For this semi-empirical calculation we took the experimental Raman spectra obtained for the two segments, $\mathrm{SiGe}$ and $\mathrm{Si}$ respectively, in regions far away the influence of the $\mathrm{HE}$, while for the $\mathrm{HE}$ we took a spectrum representative of an average composition intermediate between the composition of the two segments, approximately corresponding to a $\mathrm{Ge}$ fraction of 0.04 . Using these spectra weighted by the corresponding laser beam intensities calculated according to the data of Fig.4, we calculated the convolution spectra for different positions of the laser beam across the $\mathrm{HE}$, and for different HE widths. The calculated spectra were then compared to the experimental spectra; so the width of the HE can be estimated from the best fitting. The fitting is shown in Fig. 5a for a pure Au droplet catalysts, the best fitting is obtained for an $\mathrm{HE}$ width of $\approx 60 \mathrm{~nm}$, which is slightly larger than the NW diameter $(\approx 40 \mathrm{~nm})$. Also the effect of the resonance was considered; the spectra calculated with and without the resonance effect on the $\mathrm{HE}$ are shown in Fig.5a, the best fit to the experimental spectrum is obtained when the resonance is considered. The resonance effect in the HE is a consequence of the stronger resonance in the $\mathrm{Si}$ segment as compared to the $\mathrm{SiGe}$ segment; the resonance depends on the NW composition and diameter, the laser wavelength, and the substrate; therefore, the Raman signal must be different for two NW segments depending on all those factors.

The results obtained for a NW grown with $\mathrm{AuGa}(90 \% \mathrm{Ga})$ catalysts are shown in Fig. $5 b$. The best fitt the experimental spectrum was found for an HE width $\approx 16 \mathrm{~nm}$, which was substantially lower than the NW diameter $(\approx 40 \mathrm{~nm})$, in agreement with the results reported by other authors from STEM measurements for trailing HEs [2,3]. 

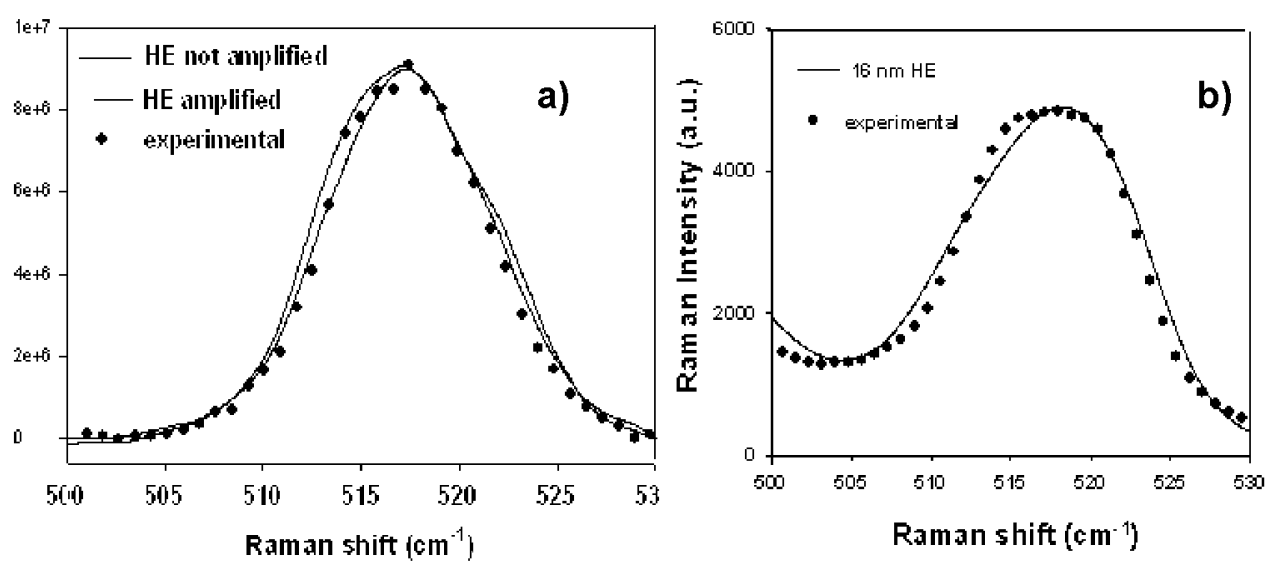

Fig.5. a) Raman spectra of the HE ( $60 \mathrm{~nm}$ width) region of a NW grown with pure Au catalysts, calculated ( full lines), with and without the optical resonance effect, and experimental (symbols). b) Calculated and experimental Raman spectra of the $\mathrm{HE}$ region for a NW grown with $\mathrm{GaAu}(90 \% \mathrm{Ga})$ catalysts, the best fitting is reached for an $\mathrm{HE}$ width of $\approx 16 \mathrm{~nm}$.

\section{Summary}

MicroRaman spectroscopy can be used to study the trailing $\mathrm{HE} \mathrm{SiGe} / \mathrm{Si}$ axial heterostructured NWs. The Raman analysis permits to estimate the HE width for NWs grown with alloyed AuGa droplet catalysts with different $\mathrm{Ga}$ concentrations, for the trailing $\mathrm{HE}$, the lower solubility of Ge in Ga reduces significantly the reservoir effect narrowing the $\mathrm{HE}$ with respect to NWs grown with pure Au catalysts.

\section{Acknowledgments}

This work was funded by the Spanish Government (Grant: MAT2010-20441-C0201-02) and Junta de Castilla y León (VA293U13).

\section{References}

1.P.Periwal, N.V.Sibirev, G. Patriarche, B.Salem, F. Bassani, V.G. Dubrovskii, T. Baron; NanoLett. 14, 5140 (2014)

2.T. E. Clark, P. Nimmatoori, K. K. Lew, L. Pan, J. M. Redwing, E. C. Dickey, Nano Lett. 8, 1246 (2008).

3.D.E. Perea, N.Li, R.M. Dickerson, A. Misra, S.T.Picraux; NanoLett. 11, 3117 (2011)

4.A. Rodríguez, T. Rodríguez, C. Ballesteros, J. Jiménez; Mat. Res. Soc. Symp. Proc. 1510, DD06-05 (2013)

5.J. Anaya, A.Torres, A. Martín-Martín, J. Souto, J. Jiménez, A. Rodríguez, T. Rodríguez; Appl. Phys. A 113, 167 (2013)

6.L.Cao, B.Nabet, J.E.Spanier; Phys. Rev. Lett. 96, 157402 (2006)

7.J.Anaya, J.Jimenez, A. Rodríguez, T. Rodríguez; Mat. Res. Soc. Symp. Proc. 1627, DOI : $10.1557 /$ opl.2014.250 (2014) 